\title{
Dynamic microenvironment and multiple damaged tissue regeneration in a de novo and synchronized manner
}

\author{
Biao Cheng ${ }^{1,2} \&$ Xiaobing $\mathrm{Fu}^{1 *}$ \\ ${ }^{1}$ College of Life Sciences, Medical College of PLA, the General Hospital of PLA, Beijing 100853, China; \\ ${ }^{2}$ The Key Laboratory of Trauma Treatment \& Tissue Repair of Tropical Area, PLA, 510010, China
}

Received November 15, 2016; accepted November 21, 2016; published online November 30, 2016

Citation: Cheng, B., and Fu, X. (2016). Dynamic microenvironment and multiple damaged tissue regeneration in a de novo and synchronized manner. Sci China Life Sci 59, 1332-1334. doi: 10.1007/s11427-016-0352-1

Regenerative medicine has rapidly developed over the past decade and created new opportunities to repair or replace tissue or organ function lost because of congenital defects, age, diseases, or serious damage (Cheng et al., 2016a; Cheng et al., 2016b). Regenerative medicine strategies include the transplantation of bioactive factors, stem cells, or biomaterials, even the induced regeneration in a de novo, depending on the application (Fu, 2014a; Huang and Fu, 2014). However, there are several limitations to the use of regenerative medicine in the clinic with respect to using stem cells and biomaterials.

The effects of bioactive factors, stem cells homing, proliferation, and directional differentiation, as well as tissue engineering material-induced inflammation, immune regulation, temporary survival, degradation, and absorption depend on the microenvironment state. Intercellular and intracellular complex cascade signaling pathways affected by the environment may determine stem cells behavior and fate (Lane et al., 2014).

Stem cell development is closely related to the microenvironment (natural stem cell niche), which provides mechanical, chemical, and topological cues and initiates a series of complex signaling events to determine stem cell fate (mitotic dormancy, self-renewal, or directional differentiation into a specific lineage). Recent studies have focused on engineering the microenvironment, which contains biological mechanics, architecture, and biological materials, modi-

*Corresponding author (email: fuxiaobing@vip.sina.com) fies bionic elements such as proteins or peptides, controls the release of growth factors, and can be used to cultivate different cell types or genetically modify cells (Badylak, 2016). Studies of both natural and engineered environments to examine stem cell regulation may also provide a basis for regenerative medicine translation.

Stem cells are grown in a three-dimensional (3D) scaffold, where both stem cells and biomaterials play key roles in de novo tissue regeneration (Ayala et al., 2015). Biomaterial effects, deriving from the material physical structure, local chemistry, and mechanical properties, have been utilized to control stem cells proliferation and differentiation. Biomaterials with various chemical compositions and physical properties such as stress, mechanical strength, topology, degradation, and vascularization, are useful for regulating tissue regeneration. Recent studies have focused on controlling the biological response and stem cells differentiation, using the extracellular environment. The microenvironment may maintain or change the function and behavior of stem cells and activate or inhibit biochemical and physical signals to promote or interfere with tissue repair and regeneration after the implantation of biomaterials (Huang et al., 2016a).

Scaffolds can control nutrient and oxygen availability while providing a physical matrix to support stem cells in tissue regeneration. Several physical factors in the stem cells microenvironment, such as topography and porosity, among others, may influence its fate, such as cellular phenotypes, growth rate, migration direction, and directional 
differentiation (Ravichandran et al., 2016; Thompson and Chan, 2016). Natural and engineered stem cell microenvironments present specific architectures, or spatial arrangements, that help regulate stem cell behavior.

Technical innovations may provide new strategies for tissue engineering. 3D printed pre-vascularized stem cells can enhance the therapeutic success of tissue repair by promoting rapid revascularization after implantation (Jang et al., 2016).

$3 \mathrm{D}$ cell printing is a promising technology for producing precisely controlled 3D tissues or organs by mimicking both the outer shape and inner architecture of native tissues. A previous study applied imprinting technology to create functional, in vitro cell-laden $3 \mathrm{D}$ extracellular matrix mimics (3D-ECM) with composite hydrogels based on gelatin and sodium alginate because of their chemical and structural similarities to ECM components. The designed 3D imprinting constructs showed high cell proliferation, improving the therapeutic effects after implanting the 3D printed tissue construct (Huang et al., 2016b). In addition, biomaterial scaffolds with a Nan fibrous structure mimic the collagen physical structure and enhanced cell/matrix interactions. Niño-technologies must be further advanced to enable more precise control over the biophysics of 3D scaffolds. The 3D shapes of many scaffolds cannot be controlled because of the limitations inherent to the methods (Zhang et al., 2014). These limitations may be overcome by combining phase separation techniques and computer assisted design and computer assisted manufacture techniques (Tang et al., 2014).

Modifications to the surface topography of biomaterials have been shown to affect inflammation and even the angiogenesis of regenerative tissues (Hotchkiss et al., 2016).

All implanted biomaterials may induce an initial acute inflammatory response, which can progress to a complex, chronic inflammation, leading to an immune response referred to as the host foreign body reaction (Aamodt and Grainger, 2016). Sometimes, regeneration activity can be inhibited after stem cells and biomaterial implantation into the body because of the hostile microenvironment at the injured sites. However, in some cases, immune cells can promote stem cells function. New biomaterials have shown minimum localized immune responses, indicating that they can be used in tissue engineering applications. They may show the high biocompatibility of Nan composites and the potential for growth factors induced soft and hard tissue regeneration (Paul et al., 2016).

The ECM represents a highly dynamic and versatile environment. All components of the ECM are degradable and subject to modification, which may affect the microenvironment. Some new implant materials have been used for physical and chemical modification to elicit a host response that can be sustained until the foreign material is removed or degraded (Keane and Badylak, 2015).

To orchestrate, support, and respond to the dynamic pro- cesses occurring during organ regeneration, the dynamic stem cell microenvironment is important ( $\mathrm{Li}$ et al., 2014) . Current demands and challenges in the field of regenerative medicine include multiple-damaged tissue regeneration in a de novo and synchronized manner, which will benefit tissue regeneration for various purposes $(\mathrm{Fu}, 2014 \mathrm{~b})$. Studies are focused on designing a bionic artificial extracellular matrix, reconstructing the optimal physical structure, and controlling physical and chemical properties beneficial to local regeneration. At the in vitro (cellular, molecular) and in vivo (animal, human body) levels, it is necessary to identify functions and interaction mechanisms among stem cells, biomaterials, and the microenvironment while considering the following points.

First, extensive effort has been dedicated to understanding the molecular mechanisms underlying stem cells fate to better control the homogeneous differentiation of stem cells prior to transplantation, which is critical for tissue formation.

Second, systematic studies have examined the interaction mechanism and signal transduction pathways between the microenvironment and regenerative cells. It is necessary to explore the biological basis of extracellular matrix in tissue development in detail and identify key regulatory proteins during the process of regeneration.

Third, studies should focus on regulating the material-dependent inflammatory response and immune response after biomaterials are implanted into the body, followed by the temporary survival, degradation, and absorption of the material. Biomaterial vascularization plays important roles in influencing the dynamic environment during degradation and regeneration.

Fourth, it is necessary to identify or develop "ideal" biological materials in accordance with the dynamic microenvironment of regeneration, which may help to accelerate tissue repair and organ formation to achieve regeneration. In addition to the use of advanced biomaterials, the fate of stem cells will be more precisely controlled.

Compliance and ethics The author(s) declare that they have no conflict of interest.

Acknowledgements This work was supported in part by the National Nature Science Foundation of China (81121004, 81230041, 81171798, 81171812, 81272105, 81671924), the National Basic Science and Development Programme (2012CB518105), and the National Science and Technology Major Project (2011ZXJ07104B-03B).

Aamodt, J.M., and Grainger, D.W. (2016). Extracellular matrix-based biomaterial scaffolds and the host response. Biomaterials 86, 68-82.

Ayala, P., Caves, J., Dai, E., Siraj, L., Liu, L., Chaudhuri, O., Haller, C.A., Mooney, D.J., and Chaikof, E.L. (2015). Engineered composite fascia for stem cell therapy in tissue repair applications. Acta Biomater 26, $1-12$.

Badylak, S.F. (2016). A scaffold immune microenvironment. Science 352, 298.

Cheng, B., Lu, S., and Fu, X. (2016a). Regenerative medicine in China: 
demands, capacity, and regulation. Burns Trauma 4, 24.

Cheng, B., Lu, S.L., and Fu, X. (2016b). Regenerative medicine in China: main progress in different fields. Mil Med Res 3, 24.

$\mathrm{Fu}, \mathrm{X}$. (2014a). Regenerative medicine research in China: from basic research to clinical practice. Sci China Life Sci 57, 155-156.

Fu, X. (2014b). Military medicine in China: old topic, new concept. Mil Med Res 1, 2.

Hotchkiss, K.M., Reddy, G.B., Hyzy, S.L., Schwartz, Z., Boyan, B.D., and Olivares-Navarrete, R. (2016). Titanium surface characteristics, including topography and wettability, alter macrophage activation. Acta Biomater 31, 425-434.

Huang, C., Du, Y., Nabzdyk, C.S., Ogawa, R., Koyama, T., Orgill, D.P., and Fu, X. (2016a). Regeneration of hair and other skin appendages: A microenvironment-centric view. Wound Repair Regen 24, 759-766.

Huang, S., and Fu, X. (2014). Stem cell therapies and regenerative medicine in China. Sci China Life Sci 57, 157-161.

Huang, S., Yao, B., Xie, J., and Fu, X. (2016b). 3D bioprinted extracellular matrix mimics facilitate directed differentiation of epithelial progenitors for sweat gland regeneration. Acta Biomater 32, 170-177.

Jang, J., Park, H.J., Kim, S.W., Kim, H., Park, J.Y., Na, S.J., Kim, H.J., Park, M.N., Choi, S.H., Park, S.H., Kim, S.W., Kwon, S.M., Kim, P.J., and Cho, D.W. (2016). 3D printed complex tissue construct using stem cell-laden decellularized extracellular matrix bioinks for cardiac repair. Biomaterials 112, 264-274.

Keane, T.J., and Badylak, S.F. (2015). The host response to allogeneic and xenogeneic biological scaffold materials. J Tissue Eng Regen Med 9, 504-511

Lane, S.W., Williams, D.A., and Watt, F.M. (2014). Modulating the stem cell niche for tissue regeneration. Nat Biotechnol 32, 795-803.

Li, M., Ti, D., Han, W., and Fu, X. (2014). Microenvironment-induced myofibroblast-like conversion of engrafted keratinocytes. Sci China Life Sci 57, 209-220.

Paul, A., Manoharan, V., Krafft, D., Assmann, A., Uquillas, J.A., Shin, S.R., Hasan, A., Hussain, M.A., Memic, A., Gaharwar, A.K., and Khademhosseini, A. (2016). Nanoengineered biomimetic hydrogels for guiding human stem cell osteogenesis in three dimensional microenvironments. J Mater Chem B Mater Biol Med 4, 3544-3554.

Ravichandran, S., Okawa, S., Martinez Arbas, S., and Del Sol, A. (2016). A systems biology approach to identify niche determinants of cellular phenotypes. Stem Cell Res 17, 406-412.

Tang, Y., Zhao, Y., Wang, X., and Lin, T. (2014). Layer-by-layer assembly of silica nanoparticles on 3D fibrous scaffolds: enhancement of osteoblast cell adhesion, proliferation, and differentiation. J Biomed Mater Res A 102, 3803-3812.

Thompson, R., and Chan, C. (2016). Signal transduction of the physical environment in the neural differentiation of stem cells. Technology 4 , $1-8$.

Zhang, L., Zhou, Y., Li, G., Zhao, Y., Gu, X., and Yang, Y. (2014). Nanoparticle mediated controlled delivery of dual growth factors. Sci China Life Sci 57, 256-262.

Open Access This article is distributed under the terms of the Creative Commons Attribution License which permits any use, distribution, and reproduction in any medium, provided the original author(s) and source are credited. 